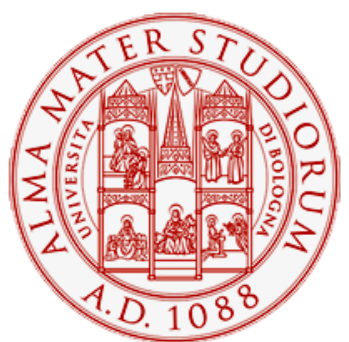

Alma Mater Studiorum - Università di Bologna DEPARTMENT OF ECONOMICS

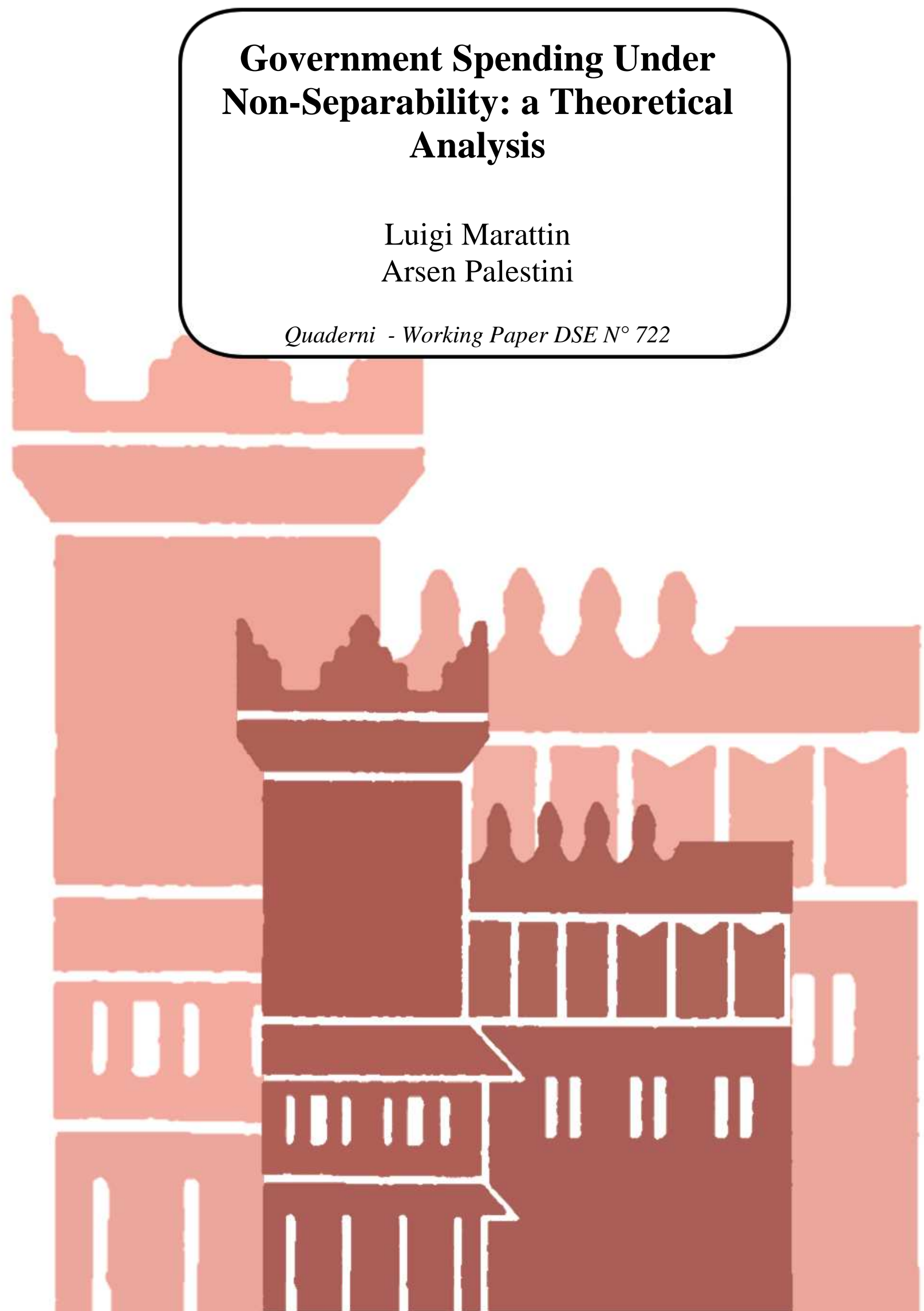




\title{
Government Spending Under Non-Separability: a Theoretical Analysis
}

\author{
Luigi Marattin \\ Department of Economics, University of Bologna \\ Strada Maggiore 45, 40125 Bologna, Italy \\ luigi.marattin@unibo.it \\ Arsen Palestini \\ MEMOTEF, Sapienza University of Rome \\ Via del Castro Laurenziano 9, 00161 Rome, Italy \\ arsen.palestini@uniroma1.it
}

22nd April 2012

\begin{abstract}
In this paper we derive analytic implicit form conditions for the qualitative analysis of government spending multipliers and the optimal level of government spending in presence of non-separability between private and public components of aggregate demand. Using the simplest neo-classical flexible price model with no capital accumulation, we show that Edgeworth dependence is not a suitable condition to automatically assess the signs of the consumption and income multipliers, for which a more complex analysis must be carried out. We propose a detailed investigation of the form and the characteristics of the involved utility functions, which are crucial to such evaluation. We also show that if Edgeworth complementarity is strong enough, a public spending stimulus can raise at the same time private consumption and real activity. In order to reconcile our general framework with existing literature, we discuss recent examples of non-separable functional forms from the standpoint of our results, and argue that their consistency relies on specific assumptions about steady- state points.
\end{abstract}




\section{Introduction}

How can economic theory rationalize the link between non-separable public and private consumption and the magnitude of government spending multipliers? After the massive fiscal stimulus in 2009, there has been a renewed interest towards theoretical and empirical investigations on the magnitude of government spending multipliers (Hall 2009, Christiano et al. 2009, Corsetti et al. 2009). This policy-led research interest builds on a consolidated theoretical effort aimed at reconciling models'predictions with the observed government spending's impact on real activity and private consumption.

Starting from the contribution by Blanchard and Perotti (2002), empirical evidence ${ }^{1}$ has reported the existence of a positive private consumption multiplier of government spending. This prediction is at odds with the standard neoclassical framework (Baxter and King 1993) and requires special assumptions - such as a high share of creditconstrained agents (Coenen and Straub 2005, Galì et al 2007)- in the New Keynesian literature. Recently, a number of contributions started working on the preferences's structure in order to rationalize the existence of a positive consumption multiplier. This effort includes papers on consumption-hours complementarity (Linneman 2006,Perotti and Monacelli 2008, Bilbiie 2009) and on consumption-public spending complementarity (Linneman and Schabert 2004, Ganelli and Tervala 2009). Guo and Harrison (2006) showed how Edgeworth complementarity in a balanced-budget policy failed to rule out indeterminacy effects. More recently, authors such as Hall (2009) and Woodford (2011) began to explore the core of the analytical issue and to derive expressions for the multipliers under different market structures.

This paper builds on these two last contributions and delves into the analytical nature of the problem. Basing on the current vacancy of a global theoretical approach to the link between public expenditure utility and multipliers' qualitative evaluation, we will provide a complete discussion on this topic. Particularly, we intend to provide a general framework in which the characteristics and the properties of the involved utility functions are closely related to the behaviour of the multipliers, showing the strong dependence between the involved functional forms and the resulting policy implications.

The usual approach consists in choosing a bundle function involving private consumption $C$ and public expenditure $G$ as utility function's arguments. For example, the following bundle functions are respectively borrowed from Ganelli and Tervala (2009) and Linnemann and Schabert (2004):

$$
B_{1}(C, G)=C+\alpha G, \quad B_{2}(C, G)=\left[\theta C^{\frac{v-1}{v}}+(1-\theta) G^{\frac{v-1}{v}}\right]^{\frac{v}{v-1}},
$$

whose related utility functions are:

$$
u_{1}\left(B_{1}(C, G)\right)=\ln \left(B_{1}(C, G)\right) \quad u_{2}\left(B_{2}(C, G)\right)=\frac{\left(B_{2}(C, G)\right)^{1-\gamma}}{1-\gamma} .
$$

In our setup we will generalize the analysis of multipliers and of Edgeworth complementarity/substitutability to each possible bundle function and utility function. We

\footnotetext{
${ }^{1}$ Among others, Fatas and Mihov 2001, Garcia and Ramajo 2005, Galì et al 2007, Ramey 2011.
} 
will determine the explicit formulas for the consumption and income multiplier without applying any log-linearization around stationary points; namely we will deal with representations of multipliers with no restrictions on the plane, except for the subsets where such formulas are not well defined. Furthermore, we use our framework to gain a better understanding of the welfare effects of public spending when the latter has non-trivial general equilibrium effects.

Our main results can be summarized as follows.

1) Edgeworth complementarity and substitutability are neither necessary nor sufficient conditions to generate - respectively - positive and negative consumption multiplier. In fact, their signs strongly depend on the characteristics of the bundle and of the utility functions, including the Arrow-Pratt measure of risk aversion of both utility and disutility functions.

2) The output multiplier is positive if marginal utility of income is positively affected by public spending (this can also occur under Edgeworth substitutability), but this is not enough: we need precise conditions on the concavity of the bundle-function.

3) A government spending stimulus can raise at the same time private consumption and output if the Edgeworth complementarity effect between private and public spending is greater than the rate of growth of marginal disutility of producing an additional unit of output.

4) The optimal level of public expenditure is decreasing in the intensity of the Edgeworth dependence, regardless of its sign.

As a support for our general analysis on multipliers, we will also provide evidence that the use of specific explicit form preference specification - along with loglinearization around specific and convenient points - is not internally consistent if applied without reference to such general theory.

This paper is organized as follows. Section 2 describes the setup of the model, the notation we employ and recalls the basic mechanism in place to determine government spending's impact in a perfectly competitive economy. In Section 3 we derive analytical expressions for the multipliers under the standard case of additive-separability between private consumption $C$ and public spending $G$. Section 4 and 5 are devoted to the derivation and discussion of signs and magnitudes of - respectively - consumption and output multiplier in a non-separability general framework. In Section 6 we verify the conditions under which both multipliers can be positive, thereby determining an expansionary effect of public spending on consumption and real activity, as often observed in empirical analysis. In Section 7 we perform a welfare analysis, deriving the conditions for the optimal level of government spending. In order to verify the general validity and consistency of our conditions, Section 8 considers two attempts to model Edgeworth dependence with two different functional forms taken from the recent literature (Ganelli and Tervala 2009, Linneman and Schabert 2004). Section 9 concludes and suggests future research directions. 


\section{The setup}

Referring to time $t \geq 0$, we will use the following notation:

- $C_{t}$ : level of private consumption;

- $G_{t}$ : flow of public expenditure;

- $H_{t}$ : amount of hours worked;

- $Y_{t}$ : quantity of the single and homogeneous good produced in the economy;

- $T_{t}$ : lump-sum taxes levied on consumers;

- $W_{t}$ : nominal hourly wage rate;

- $P_{t}$ : nominal price of good $Y_{t}$

A representative firm owned by households produces a single homogenous good $Y_{t}$ that can be privately or publicly consumed, according to a technology where hours worked are the only variable input and capital is fixed and normalized to one. So in each period:

$$
\begin{aligned}
Y_{t} & =f\left(H_{t}\right) \\
Y_{t} & =C_{t}+G_{t}
\end{aligned}
$$

where $f^{\prime}(\cdot)>0$ and $f^{\prime \prime}(\cdot)<0$ and the exogenous flow of real government expenditure $G_{t}$ is financed by the government by levying lump-sum taxes $T_{t}$ on consumers.

Households receive a nominal payment $W_{t}$ for every supplied hour; they do not save, so that their budget constraint in nominal terms is simply:

$$
P_{t} C_{t}+P_{t} T_{t}=W_{t} H_{t}
$$

We will denote with $U(\cdot)$ the utility function to be maximized by the representative household.

The above set-up describes a perfectly competitive flexible price economy with lump-sum taxation. In such a setting, an increase in government spending increases the net present value of taxation ${ }^{2}$, thereby reducing consumers' intertemporal budget constraints. The resulting negative income effect reduces the demand for both goods (consumption and leisure), provided that they are normal: the former causes the negative consumption multiplier, the latter - by increasing labor supply - causes the positive output multiplier. In this latter case, a crucial role is clearly played by how households weight the disutility of supplying labor in their preferences structure: the higher it is so the more costly it is to supply additional labor in utility terms - the lower the labor supply outwards shift and hence the lower the output multiplier. And viceversa.

\footnotetext{
${ }^{2}$ When no features is inserted to break the Ricardian equivalence - such as distortionary taxation, liquidity constraints or finite horizons - debt-financing is irrelevant to this respect.
} 
We are now ready to analyse how this mechanism displays its effects, thereby determining the size of government spending multipliers. First we present the standard case where public spending has no effects on private consumption's marginal utility, then we move to the more interesting case of non-separability.

\section{$3 \quad$ Wasteful government expenditure $\left(u_{C G}=0\right)$}

When government spending is wasteful, the instantaneous utility function can be expressed as:

$$
U(C, H)=u\left(C_{t}\right)-v\left(H_{t}\right)
$$

where $u(\cdot) \in C^{2}$ is increasing and concave in $C_{t}$ and $v(\cdot) \in C^{2}$ is increasing and convex in $H_{t}$ :

$$
u^{\prime}>0, \quad u^{\prime \prime}<0, \quad v^{\prime}>0, \quad v^{\prime \prime}>0 .
$$

Competitive equilibrium on labor and goods market leads to Pareto efficiency conditions:

$$
\begin{aligned}
f^{\prime}\left(H_{t}\right) & =\frac{W_{t}}{P_{t}} \\
u^{\prime}\left(C_{t}\right) \frac{W_{t}}{P_{t}} & =v^{\prime}\left(H_{t}\right)
\end{aligned}
$$

Combining (5) and (6) leads to:

$$
u^{\prime}\left(C_{t}\right)=\tilde{v}^{\prime}\left(Y_{t}\right)
$$

As in Woodford (2011), we indicate with $\tilde{v}^{\prime}\left(Y_{t}\right)$ households'marginal disutility of producing one additional unit of output (as a result supplying an additional hour of labor). The function $\tilde{v}$ is formally obtained by employing the substitution $\tilde{v}\left(Y_{t}\right)=$ $v\left(f^{-1}\left(Y_{t}\right)\right)$ :

$$
\frac{v^{\prime}\left(f^{-1}\left(Y_{t}\right)\right)}{f^{\prime}\left(f^{-1}\left(Y_{t}\right)\right)}=v^{\prime}\left(f^{-1}\left(Y_{t}\right)\right) \cdot\left(f^{-1}\left(Y_{t}\right)\right)^{\prime}=\left(v\left(f^{-1}\left(Y_{t}\right)\right)\right)^{\prime}=\tilde{v}^{\prime}\left(Y_{t}\right),
$$

where' indicates the derivative with respect to $Y_{t}$.

Note that the previous relation establishes a precise condition for $\tilde{v}$ to be linear. Essentially, if we assume the non-convexity of production technology in accordance to general equilibrium theory and the non-concavity of the disutility function $v(H)$, then both $v(\cdot)$ and $f^{-1}(\cdot)$ are either linear or convex. Thus $\tilde{v}$ is linear if and only if $v$ and $f^{-1}$ are both linear.

Equation (7) gives us the Pareto-efficient equilibrium output $Y_{t}$ as a function of wasteful government spending $G_{t}$. By totally differentiating it we will recover an analytical expression for the government spending multiplier on output $\left(\frac{d Y}{d G}\right)$; as (2) holds 
at equilibrium, and government expenditure is assumed to be totally wasteful, the following condition holds:

$$
d u=0 \Longrightarrow u_{Y}(Y-G) d Y=u_{G}(Y-G) d G,
$$

where we dropped the time argument for brevity and we used, like we will do in the rest of the paper, the synthetical symbol $u_{G}$ to represent the first order partial derivative of $u$ with respect to $G$. A double index will mean a second order partial derivative. From now on, we will also lighten the notation by omitting the arguments of the involved functions.

Differentiation of (7) leads to:

$$
u_{Y Y} d Y-u_{G G} d G=\tilde{v}_{Y Y} d Y,
$$

so that the multiplier expression is ${ }^{3}$ :

$$
\frac{d Y}{d G}=\frac{u_{G G}}{u_{Y Y}-\tilde{v}_{Y Y}}
$$

Equation (9) highlights that the income multiplier is:

1. non-zero if $u(\cdot)$ is non-linear;

2. strictly positive and smaller than 1 if $u(\cdot)$ is concave and $\tilde{v}(\cdot)$ is convex, as usually assumed in economic models;

3. equal to 1 if $\tilde{v}(\cdot)$ is linear, because the labor supply response is maximized given that marginal disutility is no longer increasing.

Expressing Pareto-optimality as a function of $C$ and $G$, by the same procedure, we also achieve a formulation for the consumption multiplier:

$$
u_{C C} d C=\tilde{v}_{C C} d C+\tilde{v}_{G G} d G,
$$

implying:

$$
\frac{d C}{d G}=\frac{\tilde{v}_{G G}}{u_{C C}-\tilde{v}_{C C}}
$$

Hence, the consumption multiplier is:

1. taking values between -1 and 0 as long as $\tilde{v}(\cdot)$ is convex;

2. equal to zero if $\tilde{v}(\cdot)$ is linear;

3. equal to 1 if $u(\cdot)$ is linear.

\footnotetext{
${ }^{3}$ By the Implicit Function Theorem, this representation holds in a neighbourhood of any point on the curve determined by the equilibrium condition in the $Y-G$ plane where the denominator of (??) does not vanish. In order to avoid repeating this assumption in all the remaining cases throughout the paper, we will always consider the expressions of multipliers as well-defined, i.e. in the portions of $C-G$ and $Y-G$ planes where their denominators are not vanishing.
} 
Combining (9) and (10), the general link between the two multiplier effects of government spending can be inferred:

$$
\frac{d Y}{d G}-\frac{d C}{d G}=1 \Longleftrightarrow \frac{u_{G G}}{u_{Y Y}-\tilde{v}_{Y Y}}-\frac{\tilde{v}_{G G}}{u_{C C}-\tilde{v}_{C C}}=1 .
$$

which reminds us that in perfectly competitive models with no capital accumulation - if $v(Y)$ is convex - consumption multiplier is always negative and output multiplier is always smaller than one. This is the consequence of the neoclassical mechanism recalled in Section 2. The driving force is the negative income effect, which reduces consumers'demand for the two goods which constitute the arguments of the utility function. Not surprisingly, the extent of these retrenchments - which ultimately determine multipliers' sizes - crucially relies on the specification of functional forms, particularly second order partial derivatives. We now analyze how multipliers' analytical expressions change when government spending directly affects the marginal utility of private consumption: if the effect is positive, we are in the presence of Edgeworth complementarity, while in case of a negative effect Edgeworth substitutability is said to be in place. First we look at sign and magnitude of the consumption multiplier (Section 4), then we do the same for the output multiplier (Section 5).

\section{Edgeworth-useful expenditure $\left(u_{C G} \neq 0\right)$ : the consump- tion multiplier}

In case of non-separability between private consumption and government spending, a general specification of the instantaneous utility function is:

$$
U(C, G, H)=u[B(C, G)]-v(H),
$$

where $B(C, G)$ is a bundle function involving consumption and expenditure, is a $C^{2}$ function in both variables, $u(\cdot)$ is concave ${ }^{4}$ in private and public consumption and their mixed effects do not vanish, that is $u_{C G}=u_{G C} \neq 0$. We can formulate an expression for $u_{C G}$ involving the effects related to the bundle function, which will be quite useful in the following:

$$
u_{C G}=u_{B B} B_{C} B_{G}+u_{B} B_{C G} .
$$

Recall that $u_{C G}>0$ denotes Edgeworth complementarity, whereas $u_{C G}<0$ denotes Edgeworth substitutability.

\subsection{The formulation of $\frac{d C}{d G}$}

Applying the aforementioned change of variable and plugging the constraint (3) into the relation, we can rewrite the optimal condition:

\footnotetext{
${ }^{4}$ Note that we are not requiring concavity of both utility and the bundle, but only the concavity of the composition of the two functions. As we will see in the following, our findings apply to the formulation of Ganelli and Tervala (2009), whose bundle function is linear in consumption and expenditure.
} 


$$
u_{B}[B(C, G)] B_{C}(C, G)=\tilde{v}_{Y}(C+G)=\tilde{v}_{C}(C+G),
$$

where we also took into account the change of variable:

$$
\tilde{v}_{Y}=\tilde{v}_{C} / Y_{C}=\tilde{v}_{C}
$$

We are going to introduce the analytical expression for the consumption multiplier.

Proposition 1. Given a utility function as in (12), and $u_{C G} \neq 0$, the consumption multiplier amounts to:

$$
\frac{d C}{d G}=\frac{\tilde{v}_{C G}-u_{B B} B_{C} B_{G}-u_{B} B_{C G}}{u_{B B} B_{C}^{2}+u_{B} B_{C C}-\tilde{v}_{C C}}
$$

Proof. See Appendix.

\subsection{The sign of $\frac{d C}{d G}$}

The next propositions are meant to investigate the relationship between the sign of (15) and Edgeworth complementarity/substitutability.

Proposition 2. Suppose that

$$
B_{C C}<\frac{\tilde{v}_{C C}}{u_{B}}-\frac{u_{B B}}{u_{B}} B_{C}^{2}
$$

Then the following are true:

1. If

$$
B_{C G}<-\frac{u_{B B} B_{C} B_{G}}{u_{B}},
$$

then:

$$
u_{C G}<0, \quad \frac{d C}{d G}<0 .
$$

2. If

$$
-\frac{u_{B B} B_{C} B_{G}}{u_{B}}<B_{C G}<-\frac{u_{B B} B_{C} B_{G}}{u_{B}}+\frac{\tilde{v}_{C G}}{u_{B}},
$$

then:

$$
u_{C G}>0, \quad \frac{d C}{d G}<0 .
$$

3. If

$$
B_{C G}>-\frac{u_{B B} B_{C} B_{G}}{u_{B}}+\frac{\tilde{v}_{C G}}{u_{B}},
$$

then:

$$
u_{C G}>0, \quad \frac{d C}{d G}>0 .
$$


Proof. See Appendix.

Proposition 3. Suppose that

$$
B_{C C}>\frac{\tilde{v}_{C C}}{u_{B}}-\frac{u_{B B}}{u_{B}} B_{C}^{2} .
$$

Then the following are true:

1. If

$$
B_{C G}<-\frac{u_{B B} B_{C} B_{G}}{u_{B}},
$$

then:

$$
u_{C G}<0, \quad \frac{d C}{d G}>0 .
$$

2. If

$$
-\frac{u_{B B} B_{C} B_{G}}{u_{B}}<B_{C G}<-\frac{u_{B B} B_{C} B_{G}}{u_{B}}+\frac{\tilde{v}_{C G}}{u_{B}},
$$

then:

$$
u_{C G}>0, \quad \frac{d C}{d G}>0 .
$$

3. If

$$
B_{C G}>-\frac{u_{B B} B_{C} B_{G}}{u_{B}}+\frac{\tilde{v}_{C G}}{u_{B}},
$$

then:

$$
u_{C G}>0, \quad \frac{d C}{d G}<0 .
$$

Proof. See Appendix.

Propositions 2 and 3 are summarized in the following Figure: 


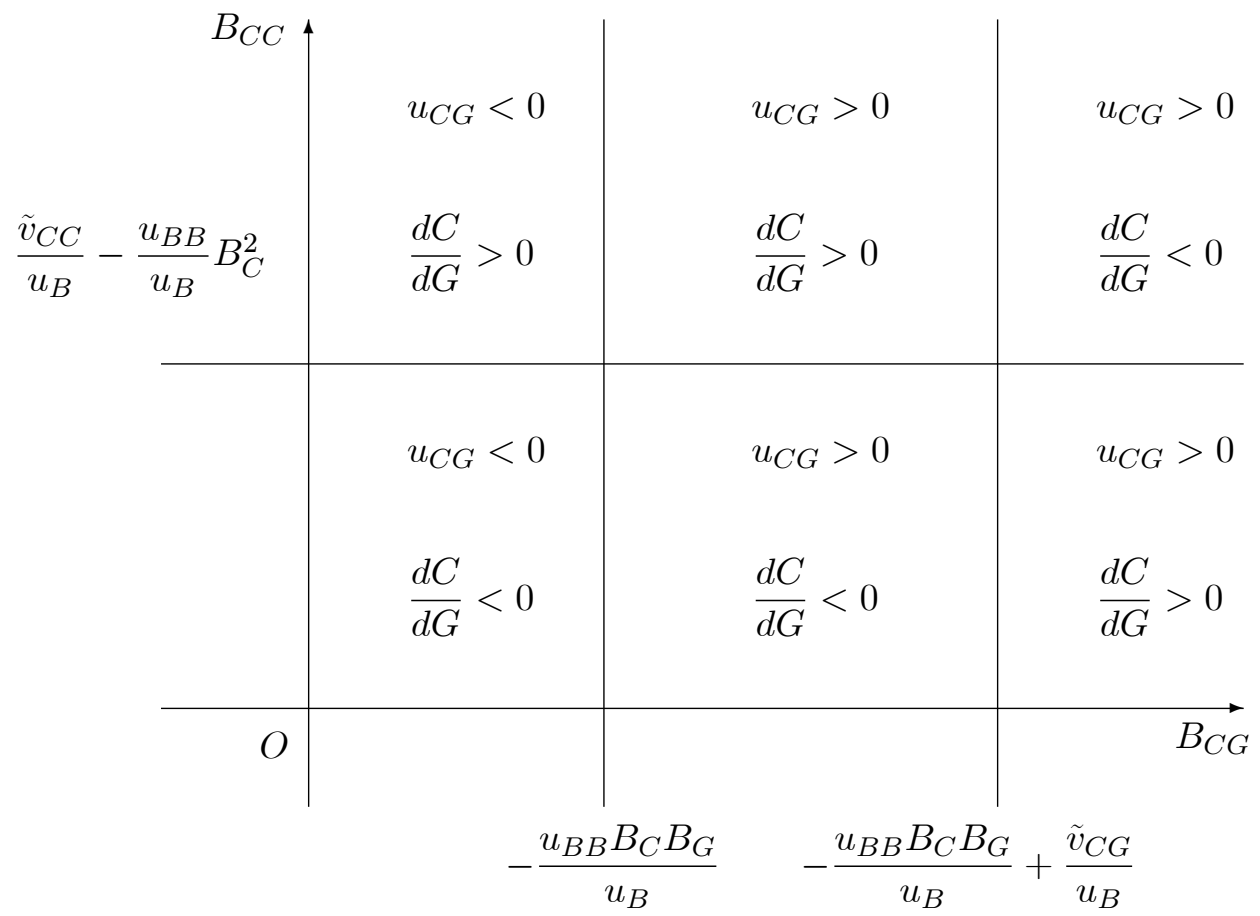

Figure 1. The six possible combinations occurring between $u_{C G}$ and $\frac{d C}{d G}$

What Propositions 2 and 3 and Figure 1 point out is that the hypothesis of Edgeworth dependence ambiguously affects the sign of the private consumption multiplier. The found thresholds depend on an absolute measure of risk aversion: if we denote with

$$
A P(u)=-\frac{u_{B B}}{u_{B}}
$$

the Arrow-Pratt index of $u$, we can remark that the threshold for $B_{C C}$ is given by $\frac{\tilde{v}_{C C}}{u_{B}}+A P(u) B_{C}^{2}$.

On the other hand, if we take into account that at equilibrium

$$
u_{B} \cdot B_{C}=u_{C}=\tilde{v}_{Y}
$$

and that

$$
\tilde{v}_{C C}=\tilde{v}_{C G}=\tilde{v}_{Y Y},
$$

then $\frac{\tilde{v}_{C G}}{u_{B}}=B_{C} \frac{\tilde{v}_{Y Y}}{\tilde{v}_{Y}}$, therefore the two relevant thresholds for $B_{C G}$ respectively are:

$$
A P(u) B_{C} B_{G} \quad \text { and } \quad B_{C}\left(A P(u) B_{G}-A P(\tilde{v})\right),
$$


highlighting the crucialness of the absolute measures of risk aversion of both utility and disutility in the sensitivity analysis.

\section{$5 \quad$ Edgeworth-useful expenditure $\left(u_{Y G} \neq 0\right)$ : the output multiplier}

Now we proceed to plug the constraint into the expression of the utility function. In order to avoid confusion, we define a new bundle function for this occasion:

$$
\mathcal{B}(Y, G)=B(C, G)_{\mid C=Y-G}=B(Y-G, G)
$$

and the related utility function

$$
U(Y, G, H)=u(\mathcal{B}(Y, G))-v(H)
$$

\subsection{The formulation of $\frac{d Y}{d G}$}

Since our aim is to write down a relation similar to (15) but just involving $Y$ and $G$, first we remark that the constraint $C=Y-G$ directly affects the first order derivative $u_{C}$, that is:

$$
\frac{\partial \mathcal{B}(Y, G)}{\partial Y}=\frac{\partial B(C, G)}{\partial C} \frac{\partial C}{\partial Y}=\frac{\partial B(C, G)}{\partial C} \Longleftrightarrow \mathcal{B}_{Y}=B_{C}
$$

Consequently, the equilibrium condition under separability (equation 7) now reads:

$$
u_{\mathcal{B}}[\mathcal{B}(Y, G)] \mathcal{B}_{Y}(Y, G)=\tilde{v}_{Y}(Y) .
$$

Before tackling the issue of the output multiplier, we have to pinpoint the other first order partial derivative of the new function:

$$
\frac{\partial \mathcal{B}(Y, G)}{\partial G}=\frac{\partial B(C, G)}{\partial C} \frac{\partial C}{\partial G}+\frac{\partial B(C, G)}{\partial G} \Longleftrightarrow \mathcal{B}_{G}=-B_{C}+B_{G} .
$$

Then, by applying the same procedure used in the calculation of the consumption multiplier and dropping the arguments as usual, we will determine the output multiplier:

Proposition 4. Given a utility function as in (16), and $u_{Y G}=u_{\mathcal{B B}} \mathcal{B}_{Y} \mathcal{B}_{G}+u_{\mathcal{B}} \mathcal{B}_{Y G} \neq 0$, the output multiplier amounts to:

$$
\frac{d Y}{d G}=-\frac{u_{\mathcal{B B}} \mathcal{B}_{G} \mathcal{B}_{Y}+u_{\mathcal{B}} \mathcal{B}_{G Y}}{u_{\mathcal{B B}} \mathcal{B}_{Y}^{2}+u_{\mathcal{B}} \mathcal{B}_{Y Y}-\tilde{v}_{Y Y}}
$$

Proof. See Appendix. 
Remark 5. The identities (18) and (15) verify the constraint $\frac{d Y}{d G}-\frac{d C}{d G}=1$. In order to ensure that, we have to take into account the following relations between derivatives:

$$
\tilde{v}_{Y Y}=\tilde{v}_{C C}=\tilde{v}_{C G}=\tilde{v}_{G G}
$$

and the properties between the derivatives of $B(\cdot)$ and $\mathcal{B}(\cdot)$. Since the denominators are equal, the difference between the multipliers is the following:

$$
\begin{gathered}
\frac{d Y}{d G}-\frac{d C}{d G}=\frac{-u_{\mathcal{B B}} \mathcal{B}_{G} \mathcal{B}_{Y}-u_{\mathcal{B}} \mathcal{B}_{G Y}-\tilde{v}_{C G}+u_{B B} B_{C} B_{G}+u_{B} B_{C G}}{u_{\mathcal{B B}} \mathcal{B}_{Y}^{2}+u_{\mathcal{B}} \mathcal{B}_{Y Y}-\tilde{v}_{Y Y}}= \\
=\frac{-u_{B B} B_{C} B_{G}+u_{B B} B_{C}^{2}-u_{B} B_{C G}+u_{B} B_{C C}-\tilde{v}_{C G}+u_{B B} B_{C} B_{G}+u_{B} B_{C G}}{u_{\mathcal{B B}} \mathcal{B}_{Y}^{2}+u_{\mathcal{B}} \mathcal{B}_{Y Y}-\tilde{v}_{Y Y}}= \\
=\frac{u_{B B} B_{C}^{2}+u_{B} B_{C C}-\tilde{v}_{C G}}{u_{\mathcal{B B}} \mathcal{B}_{Y}^{2}+u_{\mathcal{B}} \mathcal{B}_{Y Y}-\tilde{v}_{Y Y}}=1 .
\end{gathered}
$$

Remark 6. The two expressions regarding the multipliers under non-separability, i.e. (18) and (15), collapse into the expressions under separability (equations 9 and 10) if we posit $B(C, G)=C$. In fact, since all the second order partial derivatives of $B(\cdot)$ and of $\mathcal{B}(\cdot)$ vanish, $u_{C C}=u_{G G}=u_{Y Y}=u_{B B}=u_{\mathcal{B B}}$.

\subsection{The sign of $\frac{d Y}{d G}$}

The output multiplier's sign can be assessed by an analogous procedure, taking into account (19) and thus achieving the following expression:

$$
\frac{d Y}{d G}=-\frac{u_{Y G}}{u_{\mathcal{B B}} \mathcal{B}_{Y}^{2}+u_{\mathcal{B}} \mathcal{B}_{Y Y}-\tilde{v}_{Y Y}}=\frac{u_{B B} B_{C}^{2}+u_{B} B_{C C}-u_{C G}}{u_{B B} B_{C}^{2}+u_{B} B_{C C}-\tilde{v}_{C C}} .
$$

The latter double representation means that the sign of this multiplier can be discussed by evaluating either the effects of income and expenditure or the ones of consumption and expenditure, like in the case of the consumption multiplier. Following the first path, we can provide an appraisal:

Proposition 7. If either of the following holds:

1. $u_{Y G}>0$ and $\mathcal{B}_{Y Y}<\frac{\tilde{v}_{Y Y}-u_{\mathcal{B B}} \mathcal{B}_{Y}^{2}}{u_{\mathcal{B}}}$;

2. $u_{Y G}<0$ and $\mathcal{B}_{Y Y}>\frac{\tilde{v}_{Y Y}-u_{\mathcal{B B}} \mathcal{B}_{Y}^{2}}{u_{\mathcal{B}}}$; then $\frac{d Y}{d G}>0$.

Proof. Follows immediately from the expression of $\frac{d Y}{d G}$. 
Remark 8. We chose not to impose any specific assumption on the bundle function $\mathcal{B}(\cdot)$, but if we consider it as a concave function in its arguments, if $u$ is either concave or linear in $\mathcal{B}$, then the second hypothesis of Proposition 7 never holds, hence $u_{Y G}<0$ implies $\frac{d Y}{d G}<0$.

As in Subsection 4.2, also in this case the threshold for $\mathcal{B}_{Y Y}$ can be written down in terms of the Arrow-Pratt index, relying on the equilibrium identity:

$$
\frac{\tilde{v}_{Y Y}-u_{\mathcal{B B}} \mathcal{B}_{Y}^{2}}{u_{\mathcal{B}}}=-\mathcal{B}_{Y} A P(\tilde{v})+A P(u) \mathcal{B}_{Y}^{2}
$$

\section{Can government spending raise both consumption and output?}

In this Section we investigate the possibility of a government spending stimulus $d G$ raising simultaneously private consumption and real income, as reported by a number of empirical investigations summarized by Hall (2009). Before doing that, it is useful to examine some useful connections between the way public spending affects the marginal utility of - respectively- consumption $\left(u_{C G}\right)$ and income $\left(u_{Y G}\right)$. Their analytical expressions are:

$$
\begin{aligned}
u_{C G} & =u_{B B} B_{C} B_{G}+u_{B} B_{C G} \\
u_{Y G} & =u_{\mathcal{B}} \mathcal{B}_{G Y}+u_{\mathcal{B B}} \mathcal{B}_{Y} \mathcal{B}_{G}
\end{aligned}
$$

Then we exploit the relationship we derived in Subsection 5.1 between the derivatives of $B(\cdot)$ and $\mathcal{B}(\cdot)$ :

$$
\left\{\begin{array}{l}
\mathcal{B}_{G}=-B_{C}+B_{G} \\
\mathcal{B}_{Y}=B_{C} \Longrightarrow \mathcal{B}_{G Y}=B_{C G}-B_{C C}
\end{array}\right.
$$

implying:

$$
\begin{gathered}
u_{Y G}=u_{\mathcal{B}}\left(B_{C G}-B_{C C}\right)+u_{B B} B_{C}\left(-B_{C}+B_{G}\right)= \\
=u_{\mathcal{B}} B_{C G}-u_{\mathcal{B}} B_{C C}-u_{B B} B_{C}^{2}+u_{B B} B_{C} B_{G},
\end{gathered}
$$

which leads to the following identity:

$$
u_{Y G}=u_{C G}-u_{C C} .
$$

Broadly speaking, the effect of public spending on the marginal utility of income is equal to the variation between the effects of public spending and consumption on the marginal utility of consumption.

Equation 19 brings about some interesting results, of which we omit the proofs.

- If $u(\cdot)$ is concave in $C$, then $u_{Y G}>u_{C G}$. 
- If $u(\cdot)$ is concave in $C, u_{C G}>0 \Longrightarrow u_{Y G}>0$.

- If $u_{C G}<0$, then $u_{Y G}>0$ if and only if $u_{C C}<u_{C G}$.

- If $u(\cdot)$ is linear in $B$, then $u_{Y G}=u_{\mathcal{B}}\left(B_{C G}-B_{C C}\right)$.

Assuming the concavity hypothesis on $u(\cdot)$, we can stress that:

1. Under Edgeworth-complementarity $\left(u_{C G}>0\right)$ marginal utility of income is always affected by $G$ more than marginal utility of consumption is.

2. Under Edgeworth-substitutability $\left(u_{C G}<0\right)$ it is still possible to have a positive effect of $G$ on marginal utility of income, as long as $u_{C C}<u_{C G}$.

Our analysis relies on some reasonable and acknowledged assumptions, i.e. the convexity of $\tilde{v}$, entailing the inequality constraint:

$$
\tilde{v}_{Y Y}=\tilde{v}_{C C}=\tilde{v}_{C G}>0
$$

and on the positivity of the marginal contributions of consumption and expenditure to the bundle function: $B_{C}>0, B_{G}>0$. Note that these requirements are not sufficient to ensure the positivity of both marginal contributions to the other bundle function, that is only $\mathcal{B}_{Y}$ is positive, whereas $\mathcal{B}_{G}$ admits all signs.

In the following, we will focus our attention on the multipliers' behaviour under both kinds of Edgeworth dependence.

Since no capital accumulation occurs in our model, we can prove the following:

Proposition 9. Under Edgeworth complementarity, the following holds:

1. $u_{Y G}>0$;

2. if $u_{C G}>\tilde{v}_{C C}$, then $\frac{d C}{d G}$ and $\frac{d Y}{d G}$ are positive.

Proof. See Appendix.

The previous constraint $\left(u_{C G}>\tilde{v}_{C C}\right)$ has a straightforward economic interpretation. In the standard neoclassical model - absent any nominal rigidities - the output multiplier mechanism is essentially governed by the shift in households' labor supply. The size of $\tilde{v}_{C C}$ represents the velocity at which the disutility of producing one unit of output increases. Therefore, a higher $\tilde{v}_{C C}$ limits the outward shift of the labor supply curve and, consequently, the size of the output multiplier. It is therefore clear that, to ensure positivity of both multipliers, the Edgeworth complementarity effect must be strong enough to offset it.

Proposition 10. Under Edgeworth substitutability (i.e. $u_{C G}<0$ ), the following hold: 
1. $u_{Y G}>0$ if and only if $u_{C G}>u_{C C}$ if and only if

$$
\left\{\begin{array}{l}
0>\frac{d C}{d G}>-1 \\
1>\frac{d Y}{d G}>0
\end{array} ;\right.
$$

2. $u_{Y G}<0$ if and only if $u_{C G}<u_{C C}$; in this case, we have the two following cases:

- if

$$
\frac{\tilde{v}_{Y Y}-u_{\mathcal{B B}} \mathcal{B}_{Y}^{2}}{u_{\mathcal{B}}}>\mathcal{B}_{Y Y}>\frac{u_{C G}-u_{\mathcal{B B}} \mathcal{B}_{Y}^{2}}{u_{\mathcal{B}}}
$$

then

$$
\left\{\begin{array}{l}
\frac{d C}{d G}<-1 \\
\frac{d Y}{d G}<0
\end{array},\right.
$$

- if

$$
\mathcal{B}_{Y Y}>\frac{\tilde{v}_{Y Y}-u_{\mathcal{B B}} \mathcal{B}_{Y}^{2}}{u_{\mathcal{B}}}>\frac{u_{C G}-u_{\mathcal{B B}} \mathcal{B}_{Y}^{2}}{u_{\mathcal{B}}}
$$

then

$$
\left\{\begin{array}{l}
\frac{d C}{d G}>0 \\
\frac{d Y}{d G}>1
\end{array}\right.
$$

Proof. All the assertions directly follow from (??), from $u_{C G}<0<\tilde{v}_{Y Y}$ and from Proposition 7.

Consequently, if $C$ and $G$ are Edgeworth substitutes it is possible to obtain a marginal utility of income positively affected by public spending, and a positive output multiplier at the same time. What is no longer possible is the positivity of both multipliers, irrespective of the chosen bundle and utility function.

\section{Welfare analysis}

In the benchmark neoclassical model the optimal value of government expenditure is zero. This result is basically due to two considerations: i) public expenditure yields no direct utility to economic agents $i$ ) it does not deliver any indirect utility either, since it does not affect private demand's components. As a result, for a given output level, public expenditure subtracts resources from private demand and increases the 
disutility of labour supply. In other words, the "crowding-out" effect is the only one at work.

Woodford (2011) takes care of item $i$ ), and shows how this result is affected when public expenditure is inserted in a separable way into representative household's utility function. In this case, the optimal level of $G$ must satisfy:

$$
U_{G}=U_{(Y-G)}
$$

whose interpretation is straightforward: the government should spend up to the point where the marginal utility of public spending (LHS) is as high as the marginal utility of private spending (RHS). Any expenditure beyond that point would imply a situation where society would be better off if that unit of spending was used in the private sector, where the marginal utility is higher.

In this Section we are going to carry out a welfare analysis when also item ii) is faced: we are going to assume that government spending enters the utility function in a non-separable way.

Namely, we will determine the equilibrium condition of the decentralized households' problem, which will become a constraint of the planner's problem.

The decentralized problem consists in maximizing the utility function $u[B(C, G)]-$ $v(H)$ with respect to $C$ and $H$ subject to the budget constraint $C+T=W H$. First order condition of the problem is given by:

$$
W-\frac{v^{\prime}(H)}{u_{B} B_{C}}=0
$$

which, as usual, states that the marginal rate of substitution between leisure and consumption $\left(\frac{v^{\prime}(H)}{u_{B} B_{C}}\right)$ must coincide with the real wage $(W)$ when in equilibrium.

In order to find the optimal level of public expenditure, the social planner must solve:

$$
\max _{G \geq 0} u[B(C, G)]-v(H)
$$

subject to (22) and to the resource constraint (which already takes into account the production function $Y=H)$ :

$$
\begin{aligned}
& W-\frac{v^{\prime}(H)}{u_{B} B_{C}}=0 \\
& H-C-G=0
\end{aligned}
$$

Maximizing (23) subject to (24) and (25) leads to the following result, which is the non-separability version of equation $(21)$ :

Proposition 11. The optimal condition of the planner's problem is given by:

$$
U_{G}=\lambda-\mu W \frac{U_{C G}}{U_{C}}
$$


Proof. See Appendix.

In (26) $\mu$, the Lagrange multiplier associated to constraint (24), indicates the social utility value of an additional unit of wage, whereas $\lambda$, the Lagrange multiplier associated to constraint (25), indicates the social utility value of an additional unit of output.

Equation (26) determines the optimal level of public expenditure in a framework where government spending affects marginal utility of private consumption either by increasing it (Edgeworth complementarity) or by decreasing it (Edgeworth substitutability) according to the sign of $U_{C G}$.

The economic interpretation of the optimal condition is pretty straightforward. The government should spend up to the point where the marginal utility of public spending $\left(U_{G}\right)$ is equal to the net benefit stemming from its realization. In turn, the latter is equal to the social value of an additional unit of output (since the additional euro of public spending causes an increase in consumption and an overall increase in output) - $\lambda$ - minus the utility cost deriving by the increase/decrease in private consumption, which is triggered by the public expenditure stimulus. The term $\frac{U_{C G}}{U_{C}}$ gives us the extent of the movement in the marginal utility of private consumption; the resulting change in consumption must be supported by a change in labor effort, which is priced in utility terms at the marginal rate of substitution between leisure and consumption $\left(M R S_{(1-H), C}=W\right)$.

Under Edgeworth complementarity $\left(U_{C G}\right)$, a rise in $G$ increases the consumption level, thereby ruling out any possible "crowding-out" effect, since both aggregate demand components increase. Therefore output has to increase, and must be produced by households who trade leisure for consumption and pay an utility cost equal to their marginal rate of substitution $(W)$ times how much their marginal utility of consumption has increased (given by the term $\frac{U_{C G}}{U_{C}}$ ). In other words, the optimal level of public spending $\left(G^{*}\right)$ is the one in which the marginal benefit of public spending $\left(U_{G}\right)$ is equal to the social value of one additional unit of output $(\lambda)$ minus the social cost of producing it in terms of loss leisure $\left(\mu W \frac{U_{C G}}{U_{C}}\right)$. This last term, in turn, is given by the increase in marginal utility of consumption triggered by public spending $\left(=\frac{U_{C G}}{U_{C}}\right)$ multiplied by the necessary decrease in leisure which is needed in order to sustain the additional unit of consumption $(M R S=W)$, multiplied by the social utility value of wage $(\mu)$.

Under Edgeworth substitutability $\left(U_{C G}<0\right)$ the mechanism is the same, except that now private consumption decreases, so the social utility value of wage $(\mu)$ is negative. So the product $\mu W \frac{U_{C G}}{U_{C}}$ is still positive, and the expression $\lambda-\mu W \frac{U_{C G}}{U_{C}}$ still retains the same economic meaning: the difference, in utility terms, between the social value of one additional unit of output $(\lambda)$ and the social cost of producing it, where the latter incorporates the effects through private consumption.

We can note that, for $U_{G G}<0$, in both cases the optimal level of public expenditure $G^{*}$ is decreasing in the extent of Edgeworth dependence (the module of $U_{C G}$ ). Namely, 
the greater its effect on private consumption, the lower the socially desirable level of public expenditure must be.

\section{Two examples of functional forms employed in literat- ure}

In this Section we apply our previous findings to the two functional forms commonly used in the literature of non-separability to verify the consistency of our general framework and its conditions.

Our first example is borrowed from Ganelli and Tervala (2009) and is meant to show the computation of the multipliers when the bundle $B(C, G)$ is a linear combination of consumption and expenditure.

Example 12. Consider the following data:

$$
B(C, G)=C+\alpha G, \quad u(B(C, G))=\ln (B(C, G)), \quad \tilde{v}(Y)=\frac{Y^{1+\xi}}{1+\xi},
$$

where $\alpha \neq 0$ and $\xi>0$ (to ensure the required assumptions on the disutility $v(\cdot)$ ) are real parameters. Keeping in mind the constraint $Y=C+G$, we are going to reckon all the useful derivatives to determine the consumption multiplier:

$$
\begin{gathered}
u_{B}=\frac{1}{B(C, G)}, \quad u_{B B}=-\frac{1}{B^{2}(C, G)}, \quad B_{C}=1, \quad B_{G}=\alpha, \\
B_{C C}=0, \quad B_{C G}=0, \quad \tilde{v}_{C C}=\xi(C+G)^{\xi-1}, \quad \tilde{v}_{C G}=\xi(C+G)^{\xi-1} .
\end{gathered}
$$

Consequently, we have:

$$
\frac{d C}{d G}=\frac{\xi(C+G)^{\xi-1}+\frac{\alpha}{(C+\alpha G)^{2}}}{-\frac{1}{(C+\alpha G)^{2}}-\xi(C+G)^{\xi-1}} .
$$

As far as Edgeworth dependence is concerned, by the linearity of the bundle function we have that:

$$
u_{C G}=u_{B} B_{C}^{2}=-\frac{\alpha}{B^{2}(C, G)} .
$$

Keeping the value of $u_{C G}$ in mind, we can study separately two subcases:

first, a necessary and sufficient parametric condition can be inferred:

$$
\alpha>0 \Longleftrightarrow\left\{\begin{array}{l}
u_{C G}<0 \\
\frac{d C}{d G}<0
\end{array},\right.
$$

i.e., the positivity of $\alpha$ for the simultaneous Edgeworth substitutability and negativity of the consumption multiplier. ${ }^{5}$

\footnotetext{
${ }^{5}$ Note that in Ganelli and Tervala (2009) this is the rationale for the ad-hoc additive separable term $V(G)$.
} 
Being this condition solely parametric, the sign of the consumption multiplier stays negative across the whole $C-G$ plane, irrespective of the level of $C$ and/or $G$ taken into consideration.

Since the bundle function is linear $B_{C C}$ is identically zero, therefore Figure 1 collapses into 1 dimension as can be shown in the following picture:

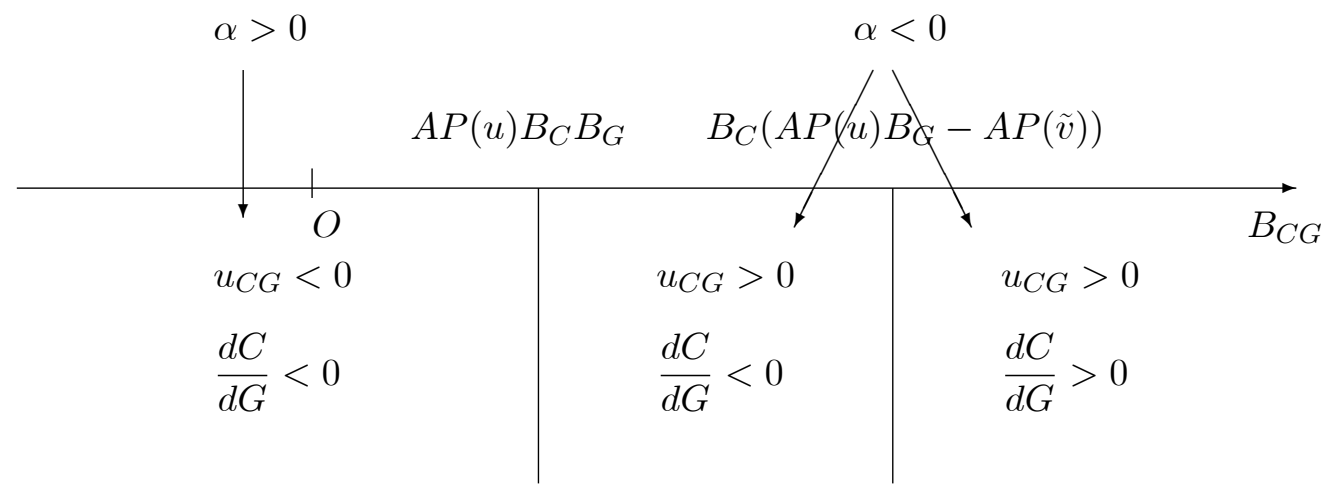

Figure 2. Qualitative analysis in Ganelli and Tervala's model

On the other hand, if $\alpha<0$, all the remaining cases may occur under complementarity, depending on the sign of the numerator of (27). Without using any log-linearization and consequently any restriction, we can evaluate the sign of (27) (not always the same) in the $C-G$ plane as if such expression were a two-parameter family of curves, where the involved coefficients were $\alpha \in(-\infty, 0)$ and $\xi \in(-\infty,-1) \cup(-1,+\infty)$.

Subsequently, we will consider the function $\mathcal{B}(Y, G)$ and its derivatives:

$$
\mathcal{B}(Y, G)=Y+(\alpha-1) G, \quad \mathcal{B}_{Y}=1, \quad \mathcal{B}_{G}=\alpha-1, \quad \mathcal{B}_{Y Y}=\mathcal{B}_{G Y}=0,
$$

so that the application of (18) yields:

$$
\frac{d Y}{d G}=\frac{\frac{\alpha-1}{[Y+(\alpha-1) G]^{2}}}{-\frac{1}{[Y+(\alpha-1) G]^{2}}-\xi(Y)^{\xi-1}}
$$

Since $u_{Y G}=\frac{1-\alpha}{\mathcal{B}^{2}(Y, G)}$, then the necessary and sufficient condition we deduce on parameter $\alpha$ is slightly different with respect to the one for (27):

$$
\alpha>1 \Longleftrightarrow\left\{\begin{array}{l}
u_{Y G}<0 \\
\frac{d Y}{d G}<0
\end{array}\right.
$$

The latter assertion confirms Ganelli and Tervala's Proposition 3, according to which $\alpha<1$ is the necessary and sufficient condition for the output multiplier to be positive, which occurs through a outward shift of the labor supply curve. 
As a further example, Linneman and Schabert (2004) employ a Constant Elasticity of Substitution aggregator between public and private consumption.

Example 13. Consider the following bundle and utility functions:

$$
B(C, G)=\left[\theta C^{\frac{v-1}{v}}+(1-\theta) G^{\frac{v-1}{v}}\right]^{\frac{v}{v-1}}, \quad u(B(C, G))=\frac{B^{1-\gamma}}{1-\gamma}
$$

and the disutility map $\tilde{v}(Y)=\frac{Y^{1+\sigma}}{1+\sigma}$, where $v \neq 1$ and $\sigma>0$.

All the relevant derivatives are computed as follows (calculations are omitted but they are available upon request):

$$
\begin{gathered}
u_{B}=[B(C, G)]^{-\gamma}, \\
B_{C B}=\theta\left(\frac{B(C, G)}{C}\right)^{\frac{1}{v}}, \\
B_{G}=(1-\theta)\left(\frac{B(C, G)}{G}\right)^{\frac{1}{v}} . \\
B_{C C}=\frac{\theta}{v}\left(\frac{B(C, G)}{C}\right)^{\frac{1}{v}}\left[-\frac{1}{C}+\frac{\theta}{B(C, G)}\left(\frac{B(C, G)}{C}\right)^{\frac{1}{v}}\right] . \\
B_{C G}=\frac{\theta(1-\theta)}{v}\left(\frac{B(C, G)}{C \cdot G}\right)^{\frac{1}{v}}[B(C, G)]^{\frac{1-v}{v}} .
\end{gathered}
$$

Consequently the derivatives of the utility functions amount to:

$$
\begin{gathered}
u_{C C}=\frac{\theta}{v} C^{-\frac{2}{v}}[B(C, G)]^{\frac{-\gamma v-v+2}{v}}\left(\theta(-\gamma v+1)-C^{\frac{1-v}{v}}[B(C, G)]^{\frac{v-1}{v}}\right) \\
u_{C G}=\theta(1-\theta)\left(-\gamma+\frac{1}{v}\right) G^{-\frac{1}{v}}\left[\theta C^{\frac{v-1}{v}}+(1-\theta) G^{\frac{v-1}{v}}\right]^{\frac{-\gamma v-v+2}{v-1}} \\
\tilde{v}_{C G}=\tilde{v}_{Y Y}=\tilde{v}_{C C}=\sigma(C+G)^{\sigma-1}
\end{gathered}
$$

Subsequently, we can plug them into (15) in order to evaluate the consumption multiplier:

$$
\frac{d C}{d G}=\frac{\sigma(C+G)^{\sigma-1}+\theta(1-\theta)(C \cdot G)^{-\frac{1}{v}}[B(C, G)]^{\frac{2}{v}-\gamma-1}\left[\gamma-\frac{1}{v}\right]}{\theta^{2} C^{-\frac{2}{v}}[B(C, G)]^{\frac{2}{v}-\gamma-1}\left[\frac{1}{v}-\gamma\right]-\frac{\theta[B(C, G)]^{\frac{1}{v}-\gamma}}{C^{\frac{1}{v}+1}}-\sigma(C+G)^{\sigma-1}} .
$$

As in Example 11, we can easily state a necessary and sufficient condition depending on the two involved parameters:

$$
v>\frac{1}{\gamma} \Longleftrightarrow\left\{\begin{array}{l}
u_{C G}<0 \\
\frac{d C}{d G}<0
\end{array}\right.
$$


i.e., on the elasticity of substitution between $C$ and $G$ and on the inverse of the degree of risk aversion $\frac{1}{\gamma}$. That is, Edgeworth substitutability is sufficient, but not necessary, to ensure the negativity of the consumption multiplier. Recalling the previous Example, this assertion holds across the $C-G$ plane too, whereas the opposite inequality can lead to different results. In Figure 3, we appraise the complementarity/substitutability and the sign of the consumption multiplier in relation to the found parametric condition, by amending Figure 1.

We can remark that all situations are admissible except the one depicted in the northwestern box, i.e. substitutability and positivity of the consumption multiplier.

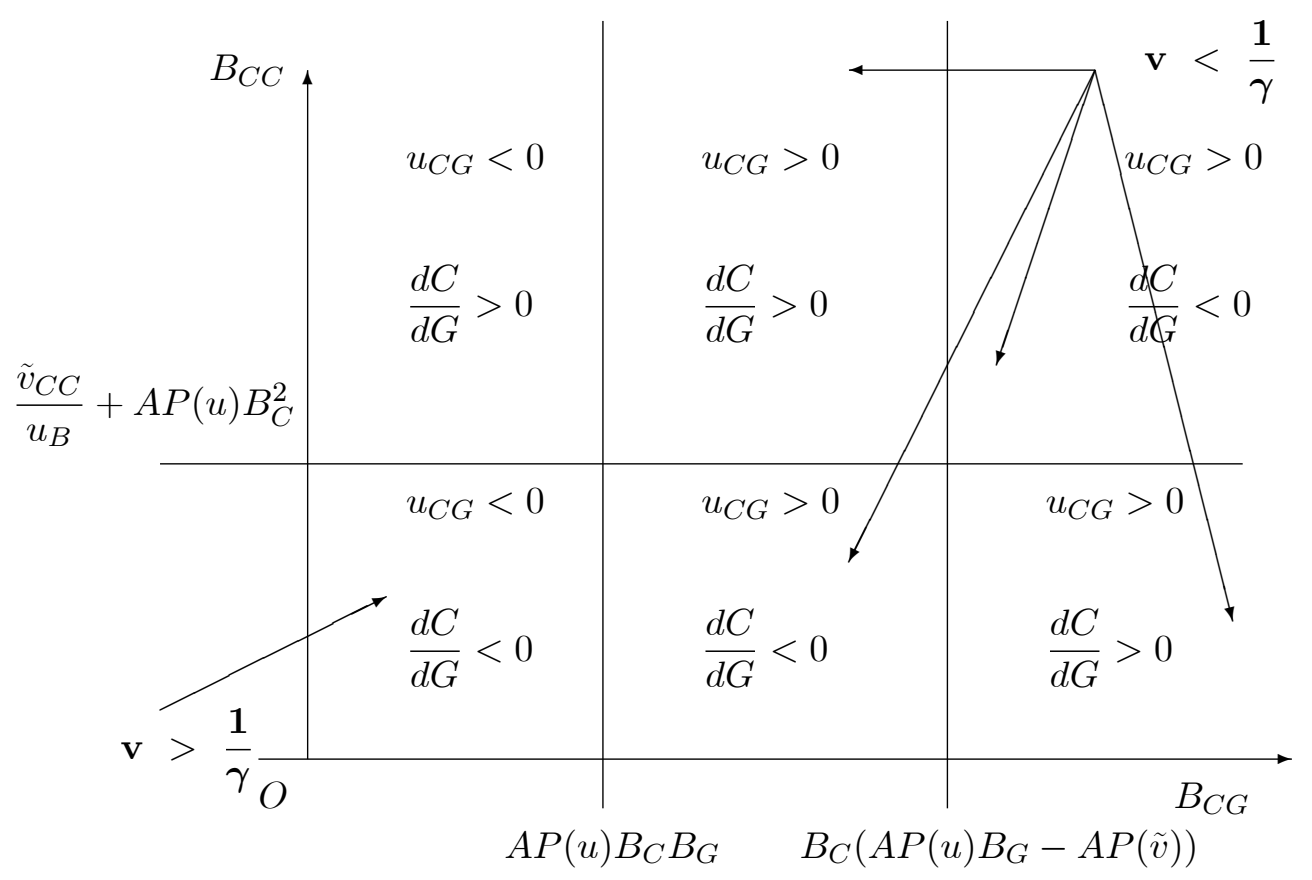

Figure 3. Qualitative analysis in Linneman and Schabert's model

By the usual position $C=Y-G$, we proceed to analyze the income multiplier. The bundle function amounts to:

$$
\mathcal{B}(Y, G)=\left[\theta(Y-G)^{\frac{v-1}{v}}+(1-\theta) G^{\frac{v-1}{v}}\right]^{\frac{v}{v-1}},
$$

and the relevant derivatives are:

$$
\begin{gathered}
\mathcal{B}_{Y}=\theta\left(\frac{\mathcal{B}(Y, G)}{(Y-G)}\right)^{\frac{1}{v}}, \quad \mathcal{B}_{G}=\mathcal{B}(Y, G)^{\frac{1}{v}}\left[(1-\theta) G^{-\frac{1}{v}}-\theta(Y-G)^{-\frac{1}{v}}\right], \\
\mathcal{B}_{G Y}=\frac{\theta}{v}\left(\frac{A(Y, G)}{Y-G}\right)^{\frac{1}{v}}\left[\mathcal{B}(Y, G)^{\frac{1}{v}-1}\left[(1-\theta) G^{-\frac{1}{v}}-\theta(Y-G)^{-\frac{1}{v}}\right]+\frac{1}{Y-G}\right], \\
\mathcal{B}_{Y Y}=\frac{\theta}{v}\left(\frac{\mathcal{B}(Y, G)}{Y-G}\right)^{\frac{1}{v}}\left[-\frac{1}{Y-G}+\frac{\theta}{\mathcal{B}(Y, G)}\left(\frac{\mathcal{B}(Y, G)}{Y-G}\right)^{\frac{1}{v}}\right] .
\end{gathered}
$$




$$
\begin{aligned}
u_{Y G}= & u_{C G}-u_{C C} \\
= & \theta(1-\theta) \frac{-\gamma v+1}{v} G^{-\frac{1}{v}}\left[\theta C^{\frac{v-1}{v}}+(1-\theta) G^{\frac{v-1}{v}}\right]^{\frac{-\gamma v-v+2}{v-1}}- \\
& \frac{\theta}{v} C^{-\frac{2}{v}}[B(C, G)]^{\frac{-\gamma v-v+2}{v}}\left(\theta(-\gamma v+1)-C^{\frac{1-v}{v}}[B(C, G)]^{\frac{v-1}{v}}\right)
\end{aligned}
$$

We can see that ambiguity emerges as early as first derivative $\mathcal{B}_{G}$ is taken into consideration; in fact, its sign ultimately determines the qualitative determination of the output multiplier.

The substitution of all previous expressions into(18) yields the following:

$$
\frac{d Y}{d G}=\frac{\frac{\theta \mathcal{B}(Y, G)^{\frac{1}{v}-\gamma}}{(Y-G)^{\frac{1}{v}}}\left[\left[-\theta(Y-G)^{-\frac{1}{v}}+(1-\theta) G^{-\frac{1}{v}}\right] \mathcal{B}(Y, G)^{\frac{1}{v}-1}\left(\gamma-\frac{1}{v}\right)-\frac{\theta}{v(Y-G)}\right]}{\frac{[\mathcal{B}(Y, G)]^{\frac{2}{v}-\gamma-1}}{(Y-G)^{\frac{2}{v}}}\left[\frac{1}{v}-\gamma\right]-\frac{\theta}{v} \frac{[\mathcal{B}(Y, G)]^{\frac{1}{v}-\gamma}}{(Y-G)^{\frac{1}{v}+1}}-\sigma Y^{\sigma-1}} .
$$

The sign of the output multiplier is ambiguous on two dimensions: the sign of the expression $\left(\gamma-\frac{1}{v}\right)$ - which, as we have just observed, determines the sign of the consumption multiplier - and the expression $\mathcal{B}_{G}$. We can easily notice that also in this case, output multiplier admits all signs according to the relative positions of steady-state points $G$ and $C$. This is why it is not rare to find analysis who apply loglinearizations around convenient points (Ganelli and Tervala (2009), for instance, loglinearize around the point $G=0$ ), so to have unambiguously positive output multipliers). However, especially when working with large-scale DSGE models where the steady-state vector is the solution to the system of first order conditions, nothing guarantees that the loglinearization is implemented around a convenient vector. Thus, our investigation defines general conditions to analyse the effects of government expenditure ${ }^{6}$.

\section{Conclusions}

The literature on fiscal policy multipliers is already very large, and still in a very active stage of development. Unfortunately, it is not always easy to compare different contributions, as fiscal multipliers can be defined in different ways depending on the purpose at hand (for example whether or not they take into account long-run effects). Moreover, empirical estimates of fiscal multipliers can be difficult to compare with those defined within more structural frameworks. With this paper, following in particular the

\footnotetext{
${ }^{6} \mathrm{~A}$ third functional form has been employed in order to model non-separability. Guo and Harrison (2006) used a specification where $u(B)$ is a CRRA utility function and $B(C, G)$ is a Cobb-Douglas bundle. We do not report such a case since, as it is well known, it is a special case of the CES specification, as it can be achieved by a logarithmic transformation of the latter.
} 
recent contribution by Woodford (2011), we wanted to frame the analysis in the easiest setup possible, the standard frictionless neoclassical model. However, we aimed at widening the traditional theoretical analysis on multipliers by explicitly taking into account the possibility that public expenditure is not simply a (often exogenous) push in aggregate demand, but indeed has per se effects on the marginal utility of private consumption. Indeed, the use of utility function specifications featuring non-separability between government expenditure and private consumption has recently gained some attention in the literature that attempts to reconcile models'predictions with observed government spending multipliers. We provided a general representation of the analytical relationships occurring between the way of modeling non-separability and the qualitative/quantitative dimensions of public spending multipliers. We obtained the following results.

First, neither Edgeworth substitutability nor Edgeworth complementarity is either necessary or sufficient to obtain a consumption multiplier with a unique sign. Crucial conditions determining the positivity or negativity can be found to be relationship between absolute measures of utility function and consumption bundle's concavities.

Second, a positive effect of government spending on the marginal utility of income is not enough to guarantee a positive output multiplier. This occurs if a certain condition on the concavity of the consumption bundle is met.

Third, if the size of Edgeworth complementarity is greater than the rate at which disutility of producing output increases, then it is possible to have both multipliers positive. In that case, a public spending stimulus raises contemporaneously private consumption and real output, as often observed in empirical analysis.

Fourth, the optimal level of public expenditure is decreasing in the intensity of the Edgeworth dependence.

This general framework has been derived in a flexible price model with competitive markets and lump-sum taxation. Once we introduce monopolistic competition, nominal price rigidities and distortionaty taxation the analytical expression of multipliers changes, as all those features play a relevant role in determining their sizes. Possible future extensions include a more complete mathematical analysis of the above theoretical contexts.

\section{Acknowledgements}

The authors would like to thank Barbara Annicchiarico, Giovanni Ganelli, Alessandra Pelloni and the audiences at the 6th Annual International Symposium on Economic Theory, Policy and Applications (25-28 July 2011, Athens) and at the SAET Annual Conference (25-30 June 2011, Faro) for valuable comments and suggestions. Usual disclaimers apply. 


\section{Appendix}

\subsection{Proof of Proposition 1}

Differentiating (14) with respect to $C$ and $G$ and dropping the arguments for brevity, the following identities hold:

$$
\left(u_{B B} B_{C}^{2}+u_{B} B_{C C}\right) d C+\left(u_{B B} B_{C} B_{G}+u_{B} B_{C G}\right) d G=\tilde{v}_{C C} d C+\tilde{v}_{C G} d G,
$$

which, after rearranging terms, entails the form (15).

\subsection{Proof of Proposition 2}

Given the hypothesis on $B_{C C}$, the expression (15) suggests that if

$$
B_{C G}>\frac{\tilde{v}_{C G}}{u_{B}}-\frac{u_{B B}}{u_{B}} B_{C} B_{G},
$$

then $\frac{d C}{d G}>0$. On the other hand, (??) implies Edgeworth-complementarity if and only if:

$$
B_{C G}>-\frac{u_{B B} B_{C} B_{G}}{u_{B}} .
$$

By previous inequalities, separating the different cases we can complete the proof.

\subsection{Proof of Proposition 3}

If

$$
B_{C C}>\frac{\tilde{v}_{C C}}{u_{B}}-\frac{u_{B B}}{u_{B}} B_{C}^{2},
$$

then by (15), $\frac{d C}{d G}>0$ if and only if

$$
B_{C G}<\frac{\tilde{v}_{C G}}{u_{B}}-\frac{u_{B B}}{u_{B}} B_{C} B_{G}
$$

Since (13) implies Edgeworth-substitutability if and only if:

$$
B_{C G}<-\frac{u_{B B} B_{C} B_{G}}{u_{B}},
$$

by taking into account all the different cases we can complete the proof.

\subsection{Proof of Proposition 4}

Differentiating (17) with respect to $Y$ and $G$ on the left-hand side and with respect to $Y$ on the right-hand side, we deduce what follows:

$$
\left(u_{\mathcal{B B}} \mathcal{B}_{Y}^{2}+u_{\mathcal{B}} \mathcal{B}_{Y Y}\right) d Y+\left(u_{\mathcal{B B}} \mathcal{B}_{G} \mathcal{B}_{Y}+u_{\mathcal{B}} \mathcal{B}_{G Y}\right) d G=\tilde{v}_{Y Y} d Y
$$

which leads to the form (18) for the output multiplier. 


\subsection{Proof of Proposition 9}

Since $u_{C G}>0$, the property $u_{Y G}>0$ follows from the concavity of $u$ with respect to consumption and from (19).

On the other hand, if $u_{C G}>\tilde{v}_{C C}$, then the output multiplier exceeds one (see Subsection 5.2). The constraint $\frac{d Y}{d G}-\frac{d C}{d G}=1$ entails that:

$$
\frac{d Y}{d G}>1 \Longleftrightarrow\left\{\begin{array}{l}
\frac{d C}{d G}>0 \\
\frac{d Y}{d G}>0
\end{array}\right.
$$

thus the second property is satisfied too.

\subsection{Proof of Proposition 11}

Calling

$$
\mathcal{L}(\cdot)=u[B(C, G)]-v(H)+\mu\left(W-\frac{v^{\prime}(H)}{u_{B} B_{C}}\right)+\lambda(H-C-G)
$$

the Lagrangian function, the FOCs of the planner's problem are:

$$
\left\{\begin{array}{l}
\frac{\partial \mathcal{L}}{\partial G}=u_{B} B_{G}+\mu v^{\prime}(H)\left[\frac{u_{B B} B_{C} B_{G}+u_{B} B_{C G}}{\left(u_{B} B_{C}\right)^{2}}\right]-\lambda=0 \\
\frac{\partial \mathcal{L}}{\partial \mu}=W-\frac{v^{\prime}(H)}{u_{B} B_{C}}=0 \\
\frac{\partial \mathcal{L}}{\partial \lambda}=H-C-G=0
\end{array}\right.
$$

where $\mu$, the Lagrange multiplier associated to the households'FOCs, indicates the social utility value of an additional unit of wage, whereas $\lambda$, the Lagrange multiplier associated to the resource constraint, indicates the social utility value of an additional unit of output.

The second and the third relation imply:

$$
v^{\prime}(H)=W u_{B} B_{C}, \quad H=C+G,
$$

then, plugging the expression of $v^{\prime}(H)$ into the first FOC, we obtain:

$$
u_{B} B_{G}+\mu W u_{B} B_{C}\left[\frac{u_{B B} B_{C} B_{G}+u_{B} B_{C G}}{\left(u_{B} B_{C}\right)^{2}}\right]-\lambda=0,
$$

and then, recalling the definitions: 


$$
\begin{aligned}
u_{B} B_{G} & =U_{G} \\
u_{B} B_{C} & =U_{C} \\
u_{B B} B_{G} B_{C}+u_{B} B_{C G} & =U_{C G}
\end{aligned}
$$

the equilibrium identity amounts to

$$
U_{G}=\lambda-\mu W \frac{U_{C G}}{U_{C}} .
$$




\section{References}

[1] Baxter, M., King, R. (1993), Fiscal Policy in General Equilibrium, American Economic Review, 83, 315-334.

[2] Bilbiie, F. (2009), Non-Separable Preferences and Frisch Labor Supply: One Solution to a Fiscal Policy Puzzle, Journal of Money, Credit and Banking, 41(2-3), 443-450.

[3] Blanchard, O., Perotti, R. (2002), An Empirical Characterization of the Dynamic Effects of Changes in Government Spending and Taxes on Output, Quarterly Journal of Economics, 117(4), 1329-68.

[4] Bouakez, H., Rebei, N. (2007), Why Does Private Consumption Rise After a Government Spending Shock?, Canadian Journal of Economics, 40(3), 954-979.

[5] Christiano, L., Eichenbaum, M., Rebelo, S. (2009), When in the Government Spending Multiplier Large?, NBER Working Paper 15394.

[6] Coenen, G., Straub, R. (2005), Does Government Spending Crowd in Private Consumption? Theory and Empirical Evidence for the Euro Area, International Finance, 8(3), 435-470.

[7] Corsetti, G., Meier A., Muller, G. (2009), Fiscal Stimulus with Spending Reversals, IMF Working paper 09/106.

[8] Fatas, A., Mihov, I. (2001), The Effects of Fiscal Policy on Consumption and Employment: Theory and Evidence, CEPR Discussion Paper 2760.

[9] Galì, J., Lopez-Salido, J.D., Vallès, J. (2007), Understanding the Effects of Goverment Expenditure on Consumption, Journal of the European Economic Association, 5(1), 227-270.

[10] Ganelli, G., Tervala, J. (2009), Can Government Spending Increase Private Consumption? The Role of Complementarity, Economic Letters, 103, 5-7.

[11] García, A., \& Ramajo, J. (2005), Fiscal policy and private consumption behaviour: the Spanish case, Empirical Economics, 30, 115-135.

[12] Guo, J.T., Harrison, S.G. (2008), Useful Government Spending and Macroeconomic (In)stability under Balanced-Budget Rules, Journal of Public Economic Theory, 10(3), 383-397.

[13] Linneman, L. (2006), The Effect of Government Spending on Private Consumption: a Puzzle?, Journal of Money, Credit and Banking, 37(7), 1715-1735.

[14] Linneman, L., Schabert, A. (2004), Can Fiscal Spending Stimulate Private Consumption?, Economic Letters, 82, 173-179. 
[15] Perotti, R., Monacelli, T. (2008), Fiscal Policy,Wealth Effects and Mark-Ups, NBER Working Paper No.14584.

[16] Ramey, V.A. (2011), Identifying Government Spending Shocks: It's All in the Timing, Quarterly Journal of Economics, 126(1), 1-50.

[17] Woodford, M. (2011), Simple Analytics of the Government Expenditure Multiplier, American Economic Journal: Macroeconomics, 3(1), 1-35. 


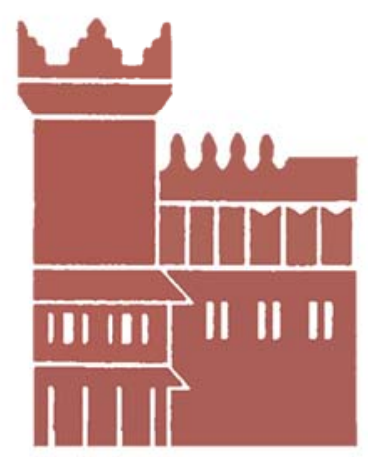

Alma Mater Studiorum - Università di Bologna DEPARTMENT OF ECONOMICS

Strada Maggiore 45

40125 Bologna - Italy

Tel. +39051 2092604

Fax +390512092664

http://www.dse.unibo.it 